\title{
Importance of glucokinase -258G/A polymorphism in Asian Indians with post-transplant and type 2 diabetes mellitus
}

\author{
Imran Ali Khan ${ }^{1,2,3}$, Kiran Kumar Vattam ${ }^{1}$, Parveen Jahan ${ }^{3}$, Qurratulain Hasan ${ }^{1,2}$, Pragna Rao ${ }^{4, *}$ \\ ${ }^{1}$ Department of Genetics and Molecular medicine, Kamineni Hospitals, Hyderabad, India; \\ ${ }^{2}$ Department of Genetics, Vasavi Medical and Research Centre, Khairathabad, Hyderabad, India; \\ ${ }^{3}$ Department of Genetics and Biotechnology, Osmania University, Tarnaka, Hyderabad, India; \\ ${ }^{4}$ Department of Biochemistry, Kasturba Medical College, Manipal University, Manipal, Karnataka, India.
}

\begin{abstract}
Summary Type 2 diabetes mellitus (T2DM) and post-transplant diabetes mellitus (PTDM) are nonsynonymous forms of diabetes. Glucokinase (GCK) plays a key role in glucose metabolism. The relationship between the $G C K$ promoter and specific types of diabetes, such as PTDM and T2DM, in the Asian Indian population is unknown. We examined the occurrence of a specific $G C K$ promoter variant $(-258 G / A)$ in patients with T2DM and PTDM. The casecontrol study enrolled 640 Asian Indian subjects, including controls $(n=250)$ and T2DM $(n=250)$, PTDM $(n=42)$, and non-post-transplant diabetes mellitus (non-PTDM) $(n=$ 98) patients. Purified Deoxyribonucleic acid (DNA) was genotyped with the polymerase chain reaction (PCR) and restriction fragment length polymorphism (RFLP) analysis. The digested PCR products were analyzed on $12 \%$ polyacrylamide gels. The anthropometric, biochemical, and clinical details of each group were documented. $G C K-258 \mathrm{G} / \mathrm{A}$ alleles and genotypes were not associated with T2DM. However, among PTDM subjects, we detected a higher frequency of heterozygotes $(\mathbf{5 2 . 4 \%})$ and a positive association with alleles/genotypes. The results suggest that the promoter region $(-258 \mathrm{G} / \mathrm{A})$ of $G C K$ plays an important role in PTDM in Asian Indians.
\end{abstract}

Keywords: GCK, -258G/A, T2DM, PTDM, Asian Indians

\section{Introduction}

Multifactorial metabolic disorders in humans include type 2 diabetes mellitus (T2DM) and post-transplant diabetes mellitus (PTDM), an irreversible form of diabetes that occurs in men and women. Impaired $\beta$-cell function and insulin resistance are key determinants of T2DM (1). The etiology of T2DM is thought to involve genetic and environmental factors, and the disease is characterized by decreased insulin secretion and insulin resistance (2). Hyperglycemia in the fasting state is one criterion that defines the disease; it can predict hard clinical endpoints in non-diabetic individuals, as well as in T2DM patients

Released online in J-STAGE as advance publication December 31, 2015.

*Address correspondence to:

Dr. Pragna Rao, Department of Biochemistry, Kasturba Medical College, Manipal University, Manipal-576104, Karnataka, India.

E-mail: drpragnarao@gmail.com when corrected (3). PTDM, also known as new-onset diabetes after transplantation (NODAT), is a frequent complication in renal transplant (RT) patients who receive immunosuppressive therapy with calcineurin inhibitors (4). Recipients with PTDM/NODAT and non-PTDM receive immunosuppressive drugs such as cyclosporine (CsA) and Tacrolimus (Tac) after renal transplantation and exhibit complications similar to those of T2DM patients in the general population, but with an accelerated rate of onset $(5,6)$. Previous investigations have focused on candidate genes expressed in pancreatic $\beta$-cells that modulate insulin secretion and resistance and predispose individuals to PTDM and T2DM in later life. One such gene, glucokinase $(G C K)$, encodes an enzyme in the glycolysis pathway that regulates glucosestimulated insulin secretion from pancreatic $\beta$-cells and metabolizes glucose in the liver (7).

Hepatic GCK activity is closely and rapidly adapted by short and long term regulatory mechanisms. Peter et al. (8) group for the first time demonstrated the positive correlation between hepatic GCK expression and liver 
triglyceride content in humans. A reduction in GCK levels or activity in $\beta$-cells could increase the glucose threshold for insulin secretion, leading to atypical hyperglycaemic status (9). The $G C K$ gene is located at chromosome 7 p15.3 and has 12 exons (10). GCK genetic variants contribute to the risk of T2DM in men and women of different ethnicities, but their frequencies in Asian Indians with PTDM and T2DM have not been examined. There are no studies that have been carried out with $-258 \mathrm{G} / \mathrm{A}$ variants in the Indian population with any of these diseases. Hence, the current study investigated whether the $-258 \mathrm{G} / \mathrm{A}$ variant in the promoter region of GCK modifies the risk of T2DM and PTDM in Asian Indians with and without a family history of T2DM.

\section{Materials and Methods}

\subsection{Ethical approval}

The study was approved by the ethics committee of Kamineni Hospitals, Hyderabad, India (KHL No. e373/07). The participants signed written informed consent forms before enrolment.

\subsection{Selection of subjects}

For this study, we recruited 640 Asian Indian subjects: 250 subjects with T2DM, 250 healthy controls, 42 subjects with PTDM, and 98 subjects without PTDM (non-PTDM). The selection of T2DM subjects, controls, and PTDM subjects and the inclusion and exclusion criteria for the specific groups are described in previous publications $(1,5,11)$. We collected $3 \mathrm{~mL}$ of serum for biochemical analysis and $2 \mathrm{~mL}$ of anticoagulated blood for mutational analysis. The anthropometric, biochemical, and clinical measurements for T2DM, PTDM, and non-PTDM cases and control subjects are described in our previous reports $(1,5,11)$.

\subsection{Mutational analysis}

DNA was routinely purified at Kamineni Hospitals using a salting out procedure $(12,13)$. The region 173 bp upstream of the $G C K$ coding region was genotyped with polymerase chain reaction (PCR) and restriction fragment length polymorphism (RFLP) analysis. Primers were designed according to previous studies (14) and synthesized by BioServe Biotechnologies (Hyderabad, India). The amplified products were digested with the AccI restriction enzyme (GTG $\left.{ }^{\downarrow} \mathrm{TAC}\right)$ for 2 hours, and the fragments were analyzed on $12 \%$ polyacrylamide gels stained with ethidium bromide (Figure 1).

\subsection{Statistical analysis}

Clinical data were expressed as the mean \pm standard deviation (SD). Differences in genotype and allele

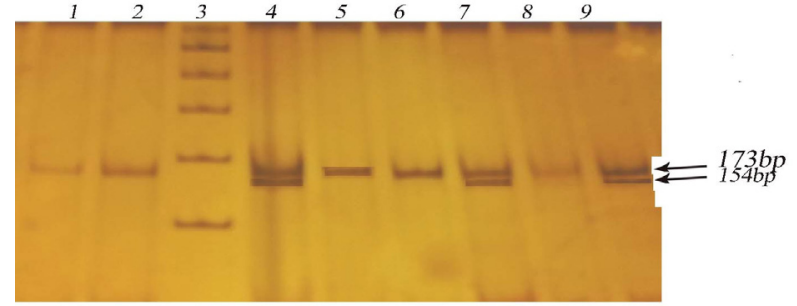

Figure 1: Digested products separated by $12 \%$ polyacrylamide gel electrophoresis for assessment of $\mathbf{- 2 5 8 G}$ / A GCK variants. Lane: 1, 2, 5, 6, 8 Homezygous GG 173bp; Lane: 3 Ladder (100bp); Lane: 4, 7, 9 Heterozygous GA 173/154bp.

frequencies were determined with the gene counting method for T2DM vs. controls and PTDM vs. nonPTDM subjects. An odds ratio (OR) with a 95\% confidence interval (CI) was calculated for the $-258 \mathrm{G} / \mathrm{A}$ mutation. The chi-square $\left(\chi^{2}\right)$ test was used to compare expected and observed frequencies of categorical variables, at a significance level $p=0.05$. The Yates correction was also used to analyze genotype frequencies. The Hardy-Weinberg equilibrium (HWE) was measured using the $\chi^{2}$ test to assess goodness of fit (15). A value of $p<0.05$ was considered to indicate a significant disequilibrium. Statistical calculations were performed with Openepi software.

\section{Results}

\subsection{Baseline characteristics of T2DM patients and controls}

The anthropometric, biochemical, and clinical characteristics of the study groups are shown in Table 1. The average age was $57.19 \pm 8.22$ years for T2DM patients and $53.93 \pm 6.32$ years for controls. The mean body mass index (BMI) was $27.5 \pm 4.1 \mathrm{~kg} / \mathrm{m}^{2}$ for T2DM cases and $25.8 \pm 3.9 \mathrm{~kg} / \mathrm{m}^{2}$ for controls. There were significant differences between T2DM patients and controls in the levels of fasting blood sugar, postlunch blood sugar, triglycerides, HDL-C, and total cholesterol $(p<0.05)$. Gender, BMI, LDL-C, and family history were not significantly associated with T2DM ( $p>0.05)$, although family history was closely associated with T2DM (58.4\%).

\subsection{Characteristics of the PTDM and non-PTDM subjects}

The characteristics of the PTDM and non-PTDM subjects are given in Table 2. Biochemical tests were used to assess PTDM in 140 RT patients; $30 \%$ of RT recipients developed PTDM. The study included 42 patients diagnosed with PTDM and 98 patients diagnosed as non-PTDM subjects. The PTDM patient group contained 30 men and 12 women, with similar mean ages, $39.39 \pm 2.12$ and $40.01 \pm 11.63$, 
Table 1. Clinical details of T2DM patients and the healthy controls

\begin{tabular}{|c|c|c|c|}
\hline Characteristics & T2DM Cases $(n=250)$ & Healthy Controls $(n=250)$ & $p$ value \\
\hline Age (Years) & $41-82(57.19 \pm 8.22)$ & $41-60(53.93 \pm 6.32)$ & 0.0003 \\
\hline Males/Females (\%) & $138(55.2 \%) / 112(44.8 \%)$ & $144(57.6 \%) / 106(42.4 \%)$ & 0.3461 \\
\hline BMI $\left(\mathrm{kg} / \mathrm{m}^{2}\right)$ & $27.5 \pm 4.1$ & $25.8 \pm 3.9$ & 0.4306 \\
\hline T2DM Interval & $13.1 \pm 6.3$ & NA & NA \\
\hline FBS (mg/dL) & $143.61 \pm 55.66$ & $93.54 \pm 12.13$ & 0.0001 \\
\hline PPBG (mg/dL) & $201.29 \pm 25.25$ & $117.29 \pm 19.07$ & 0.0001 \\
\hline $\mathrm{TG}(\mathrm{mg} / \mathrm{dL})$ & $156.42 \pm 78.97$ & $138.77 \pm 53.69$ & 0.0001 \\
\hline $\mathrm{TC}(\mathrm{mg} / \mathrm{dL})$ & $183.95 \pm 51.54$ & $175.06 \pm 33.05$ & 0.0001 \\
\hline HDL-C (mg/dL) & $88.72 \pm 23.1$ & $82.61 \pm 20.6$ & 0.01 \\
\hline LDL-C (mg/dL) & $38.76 \pm 4.4$ & $35.53 \pm 4.1$ & 0.2658 \\
\hline Family History, $n(\%)$ & $146(58.4 \%)$ & $138(55.2 \%)$ & 0.3745 \\
\hline
\end{tabular}

$\mathrm{NA}=$ Not Analyzed/ Not Applicable.

Table 2. Clinical characteristics of PTDM and non-PTDM subjects

\begin{tabular}{lccc}
\hline Baseline characteristics & $\operatorname{PTDM}(n=42)$ & non-PTDM $(n=98)$ & $p$ value \\
\hline Males / Females & $0 / 12$ & $75 / 23$ & 0.001 \\
Age: & & $39.55 \pm 10.58$ & 0.27 \\
$\quad$ a) Males (Mean \pm SD) & $39.39 \pm 12.12$ & $39.26 \pm 10.87$ & 0.58 \\
b) Females (Mean \pm SD) & $40.01 \pm 11.63$ & $66.03 \pm 12.73$ & 0.08 \\
Weight: & $62.73 \pm 15.81$ & $65.49 \pm 13.68$ & 0.09 \\
$\quad$ a) Males (Mean \pm SD) & $61.71 \pm 16.93$ & & 0.01 \\
b) Females (Mean \pm SD) & 22 & 58 & 0.02 \\
Therapy: & 20 & $201.29 \pm 76.86$ & 0.03 \\
$\quad$ a) On CsA therapy & & $3.11 \pm 1.62$ & 0.05 \\
b) On Tac therapy & $163.88 \pm 57.4$ & $1024.8 \pm 353.42$ & 0.23 \\
Dose: & $3.15 \pm 1.24$ & $8.0 \pm 3.32$ & 0.86 \\
$\quad$ a) CsA Dose (mg) & $750 \pm 299.03$ & $5.52 \pm 1.97$ & 0.02 \\
b) Tac Dose (mg) & $9.55 \pm 3.38$ & $2.98 \pm 1.49$ & 0.02 \\
Levels: & & & \\
$\quad$ a) C2 levels (ng/mL) CsA & $5.24 \pm 2.59$ & & \\
b) Trough levels (ng/mL) Tac & $3.62 \pm 1.96$ & & \\
Levels: & & & \\
$\quad$ a) C2 levels/dose of CsA & & & \\
b) Trough levels/dose of Tac & & & \\
\hline
\end{tabular}

respectively. Of the 42 patients with PTDM, 22 (54\%) were receiving cyclosporine A (CsA) treatment, whereas $20(47.6 \%)$ were receiving tacrolimus (Tac). The mean age of the PTDM patients on the date of post-transplant blood sample collection was $20.4 \pm$ 15.3 years. The t-test was performed between PTDM and non-PTDM subjects, gender, weight, subjects with cyclosporine drugs (i.e., CsA, Tac) and along with the dosage were found statistically significant $(p<0.05)$.

\subsection{Hardy-Weinberg Equilibrium}

The distributions of the genotype frequencies of the $-258 \mathrm{G} / \mathrm{A}$ variants in case and control subjects were in accordance with the HWE (Table 3). The genotypic distribution of GCK variants and their allelic frequencies in the patients (T2DM and PTDM) and controls enrolled in this study are shown in Table 4.

\subsection{Association of the $-258 \mathrm{G} / \mathrm{A}$ variant with $T 2 D M$ and PTDM}

The genotype and allele distributions in cases (T2DM
Table 3. Details of Hardy Weinberg equilibrium

\begin{tabular}{ll}
\hline Gene & Glucokinase \\
\hline SNP ID & rs1799831 \\
Chromosome & $7 \mathrm{p} 15.3$ \\
Position (Build36.3) & $44,199,142$ \\
PMID & 11112984 \\
Organism & Homosapiens \\
Alleles $1 / 2$ & $\mathrm{G} / \mathrm{A}$ \\
Risk allele & $\mathrm{A}$ \\
T2DM genotypes $(11 / 12 / 22)$ & $246 / 04 / 00$ \\
PTDM genotypes $(11 / 12 / 22)$ & $20 / 22 / 00$ \\
Non-PTDM genotypes $(11 / 12 / 22)$ & $92 / 06 / 00$ \\
Control Genotypes $(11 / 12 / 22)$ & $248 / 02 / 00$ \\
Risk allele frequency $\left(\mathrm{T} / \mathrm{P}^{*}\right)$ & $0.8 / 26.2$ \\
HWE (Chi square) $\left(\mathrm{T} / \mathrm{P}^{*}\right)$ & $0.02 / 1.73$ \\
HWE $\left(p\right.$ value) $\left(\mathrm{T} / \mathrm{P}^{*}\right)$ & $0.99 / 0.9$ \\
\hline
\end{tabular}

$\left(\mathrm{T} / \mathrm{P}^{*}\right)$ indicates T2DM/PTDM.

and PTDM) and controls (healthy controls and nonPTDM) are shown in Table 4. The genotype frequency of GG and GA was $98.4 \%$ and $1.6 \%$ in T2DM cases and $99.2 \%$ and $0.8 \%$ in controls, respectively. In the statistical analysis, we did not find any association 
Table 4. Genotype and allele distribution of -258G/A GCK variants in all cases (T2DM and PTDM) and controls (healthy controls and non-PTDM)

\begin{tabular}{|c|c|c|c|c|c|}
\hline GCK & $\mathrm{T} 2 \mathrm{DM}(n=250)$ & Controls $(n=250)$ & $\chi^{2}$ & Odds ratio ${ }^{\text {a\&b }}(95 \% \mathrm{CI})$ & $p$ value \\
\hline$G G$ & $246(98.4 \%)$ & $248(99.2 \%)$ & Reference* & - & - \\
\hline GA & $04(1.6 \%)$ & $02(0.8 \%)$ & 0.67 & $2.0(0.36,11.1)$ & 0.41 \\
\hline$A A$ & $00(00)$ & $00(00)$ & 0.001 & $1.0(0.01-51)$ & 0.31 \\
\hline$G A+A A$ & $04(1.6 \%)$ & $02(0.8 \%)$ & 0.57 & $1.8(0.38,8.6)$ & 0.70 \\
\hline$G$ & 496 (99.2\%) & 498 (99.6\%) & Reference* & - & - \\
\hline \multirow[t]{2}{*}{$A$} & $04(0.8 \%)$ & $02(0.4 \%)$ & 0.67 & $2.0(0.36,11.0)$ & 0.41 \\
\hline & $\operatorname{PTDM}(n=42)$ & Non-PTDM $(n=98)$ & $\chi^{2}$ & Odds ratio ${ }^{\text {a\&b }}(95 \% \mathrm{CI})$ & $p$ value \\
\hline$G G$ & $20(47.6 \%)$ & $92(93.9 \%)$ & Reference* $^{*}$ & - & - \\
\hline$G A$ & $22(52.4 \%)$ & $06(6.1 \%)$ & 39.04 & $16.8(6.0,46.9)$ & 0.0001 \\
\hline$A A$ & $00(00)^{*}$ & $00(00)$ & 0.66 & $4.5(0.08,234.1)$ & 0.63 \\
\hline$G A+A A$ & $22(52.4 \%)$ & $06(6.1 \%)$ & 38.35 & $15.6(5.7,42.2)$ & 0.0001 \\
\hline G & $62(0.74)$ & $190(0.97)$ & Reference* & - & - \\
\hline$A$ & $22(0.26)$ & $06(0.03)$ & 34.83 & $11.2(4.3,28.9)$ & 0.0001 \\
\hline
\end{tabular}

${ }^{\mathrm{a}-}$ Crude odds ratio $(95 \% \mathrm{CI}) ;{ }^{\text {b- }}$ odds ratio $(95 \% \mathrm{CI})$ Adjusted for Yates correction. ${ }^{*}$ Reference indicated the normal allele/genotypes i.e. GG is normal/homozygous genotype and $\mathrm{G}$ allele is wild allele.

between alleles and genotypes $(\mathrm{G}$ vs. $\mathrm{A}: \mathrm{OR}=2.0$ [95\% CI: $0.36-11.0], p=0.41$; GA vs. GG: OR $=2.0$ [95\% CI: 0.36-11.1], $p=0.41)$. Statistically significant differences in allele and genotype frequencies were observed when PTDM and non-PTDM subjects were compared (G vs. A: OR = 11.2 [95\% CI: 4.3-28.9], $p<$ 0.0001; GA vs. GG: OR = 16.8 [95\% CI: 6.0-46.9], $p<$ $0.0001)$. The dominant model also showed a significant association $(\mathrm{GA}+\mathrm{AA}$ vs. AA: $\mathrm{OR}=15.6$ [95\% CI: 5.742.2], $p<0.0001)$.

\section{Discussion}

Gene identification is important for understanding the pathophysiology of disease and for improving diagnosis, prevention, and treatment (16). We performed a casecontrol study to evaluate the association between $-258 \mathrm{G} /$ A GCK variants and PTDM and T2DM in the Asian Indian population. To our knowledge, this study is the first to find that alleles and genotypes are associated with an increased risk of PTDM $(p<0.0001)$. T2DM and PTDM are chronic disorders characterized by hyperglycemia, insulin resistance, and impaired insulin secretion due to pancreatic $\beta$-cell defects; the diseases have a strong genetic component (7). In India, 65.1 million adults have been diagnosed with T2DM (17). PTDM has become a serious health complication worldwide. Insulin is used to monitor glucose values for more than 6 months after transplantation, in PTDM subjects and lifelong without transplantation. In those with T2DM, the disease develops in the later thirties and early forties and progresses. Those with PTDM develop diabetes 3 months after RT and may later develop T2DM.

One of our goals was to investigate whether -258 $\mathrm{G} / \mathrm{A}$ variants in the promoter region of $G C K$ are associated with T2DM in the Asian Indian population. We compared T2DM cases with control subjects and found no significant differences in allele or genotype frequencies. This was the first study carried out in this population. The discrepancy between the results of the present study (T2DM vs. controls) and those of Chinese studies can be attributed to the $G C K$ gene in the Chinese population (18). Our study is consistent with three previous studies of T2DM in different races and ethnicities, which found that $G C K$ was not associated with T2DM (19-21). Our results also showed that the $-258 \mathrm{G} / \mathrm{A}$ GCK variant had no significant effect on abnormal glucose levels in T2DM patients receiving noninsulin treatment. The extent to which the $G C K$ promoter variants affect these traits might vary, possibly explaining the contrasting results. Mutations in $G C K$ can alter the reaction. Some rare exonic mutations have been investigated previously, and the role of these mutations in the pathogenesis of reduced fetal growth and in adult insulin resistance has been established. $G C K$ is associated with reduced $\beta$-cell function, impaired glucose tolerance, and T2DM (22). Yang et al. (23) performed a meta-analysis of the $-30 \mathrm{G}>\mathrm{A} G C K$ polymorphism in gestational diabetes mellitus (GDM) and T2DM and concluded that the A allele was a risk factor for GDM and T2DM among whites. Fu et al. (7) also performed meta-analysis studies of T2DM with 298,468 subjects, and the results agree with those of Yang et al. (23). Our study is not in agreement with these studies. The small sample size could be one reason.

In the comparison of PTDM vs. non-PTDM patients, promoter region variants were significantly associated with PTDM $(p<0.0001)$. Our results in PTDM subjects are in accordance with those from a study of a Korean population (24). In our study population, PTDM and GDM (unpublished data) were strongly associated with $G C K$. Given these results, we cannot conclude that mutations in GCK have no role in the Asian Indian population. The lack of any significant association between the variants and disease might be due to several 
factors, including ethnicity, low sample size, and low statistical power. Of 640 subjects, none had the AA genotype, suggesting that the AA genotype is associated with disease development.

The current study has several limitations. Firstly, we enrolled a small number of PTDM $(n=42)$, and non-PTDM $(n=98)$ subjects. Secondly, only promoter region variants were selected. Thirdly, GDM data was excluded to strengthen the study. In addition, insulin levels were not measured. Finally, biochemical data for PTDM and non-PTDM subjects were not obtained. Our study suggests that large, unbiased epidemiological studies of genetic predisposition can provide insight into the in vivo relationship between candidate genes and diseases. The reproduction of genetic association studies can be problematic because of insufficient power, multiple hypothesis testing, population stratification, source of controls, publication bias, and phenotypic heterogeneity (25).

\section{Discussion}

The findings of this study indicate that PTDM has a strong genetic association with $G C K$. Our findings also indicate that the $-258 \mathrm{G} / \mathrm{A}$ variant in the promoter region of $G C K$ is not an independent risk factor for the development of T2DM in Asian Indian subjects.

\section{Acknowledgements}

We are deeply thankful to Dr. Kamal Kiran Mukkavali, a nephrologist who assisted with the RT samples, and to Dr. Sireesha Movva, who assisted with the DNA samples for T2DM. We are grateful to ICMR for funding this work (Sanction no.5-3-8-39-2007; RHN).

\section{References}

1. Khan IA, Movva S, Shaik NA, Chava S, Jahan P, Mukkavali KK, Kamineni V, Hasan Q, Rao P. Investigation of Calpain 10 (rs2975760) gene polymorphism in Asian Indians with gestational diabetes mellitus. Meta Gene. 2014; 2:299-306.

2. Saitoh S, Shimoda T, Hamamoto Y, Nakaya Y, Nakajima S. Correlations among obesity-associated gene polymorphisms, body composition, and physical activity in patients with type 2 diabetes mellitus. Indian J Endocrinol Metab. 2015; 19:66-71.

3. Dupuis J, Langenberg C, Prokopenko I, et al. New genetic loci implicated in fasting glucose homeostasis and their impact on type 2 diabetes risk. Nat Genet. 2010; 42:105-116.

4. Tavira B, Gomez J, Diaz-Corte C, Llobet L, Ruiz-Pesini E, Ortega F, Coto E. Mitochondrial DNA haplogroups and risk of new-onset diabetes among tacrolimus-treated renal transplanted patients. Gene. 2014; 538:195-198.

5. Khan IA, Vattam KK, Jahan P, Mukkavali KK, Hasan Q, Rao P. Correlation between KCNQ1 and KCNJ11 gene polymorphisms and type 2 and post-transplant diabetes mellitus in the Asian Indian population. Genes \& Diseases. 2015; 2:276-282.

6. Ghisdal L, Van Laecke S, Abramowicz MJ, Vanholder $\mathrm{R}$, Abramowicz D. New-onset diabetes after renal transplantation: Risk assessment and management. Diabetes Care. 2012; 35:181-188.

7. Fu D, Cong X, Ma Y, Cai H, Cai M, Li D, Lv M, Yuan X, Huang Y, Lv Z. Genetic polymorphism of glucokinase on the risk of type 2 diabetes and impaired glucose regulation: Evidence based on 298,468 subjects. PLoS One. 2013; 8:e55727.

8. Peter A, Stefan N, Cegan A, Walenta M, Wagner S, Konigsrainer A, Königsrainer I, Machicao F, Schick F, Häring HU, Schleicher E. Hepatic glucokinase expression is associated with Lipogenesis and fatty livers in Humans. J Clin Endocrinol Metab. 2011; 96:E1126-E1130.

9. Naylor R, Philipson LH. Who should have genetic testing for maturity-onset diabetes of the young? Clin Endocrinol (Oxf). 2011; 75:422-426.

10. Sotos-Prieto M, Luben R, Khaw KT, Wareham NJ, Forouhi NG. The association between Mediterranean Diet Score and glucokinase regulatory protein gene variation on the markers of cardiometabolic risk: An analysis in the European Prospective Investigation into Cancer (EPIC)-Norfolk study. Br J Nutr. 2014; 112:122131.

11. Khan IA, Jahan P, Hasan Q, Rao P. Validation of the association of TCF $7 L 2$ and SLC $30 A 8$ gene polymorphisms with post-transplant diabetes mellitus in Asian Indian population. Intractable Rare Dis Res. 2015; 4:87-92.

12. Khan IA, Shaik NA, Kamineni V, Jahan P, Hasan Q, Rao P. Evaluation of gestational diabetes mellitus risk in south Indian women based on MTHFR (C677T) and FVL (G1691A) Mutations. Front Pediatr. 2015; 3:34.

13. Khan IA, Vasundhara K, Poornima S, Jahan P, Hasan Q, Rao P. Tumor necrosis factor alpha promoter polymorphism studies in pregnant women. J Reprod Health Med. 2015; 1:18-22.

14. Chiu KC, Chuang LM, Yoon C, Saad MF. Hepatic glucokinase promoter polymorphism is associated with hepatic insulin resistance in Asian Indians. BMC Genet. 2000; $1: 2$.

15. Rodriguez S, Gaunt TR, Day IN. Hardy-Weinberg equilibrium testing of biological ascertainment for Mendelian randomization studies. Am J Epidemiol. 2009; 169:505-514.

16. Butler, M, Bliss D, Drekonja D, Filice G, Rectar T, Mac Donald R, et al. Effectiveness of Early Diagnosis, Prevention, and Treatment of Clostridium difficile Infection. 2011; Report No.: 11(12)-EHC051-EF.

17. Saboo B, Reddy GC, Juneja S, Kedia AK, Manjrekar P, Rathod R, behalf of GLOBE Investigators. Effectiveness and safety of fixed dose combination of acarbose/ metformin in Indian type 2 diabetes patients: Results from observational GLOBE Study. Indian J Endocrinol Metab. 2015; 19:129-135.

18. Liu Z, Deng H, Tang WL. Mutation of GCK gene of Chinese patients with late-onset type 2 diabetes. Hunan Yi Ke Da Xue Xue Bao. 2003; 28:99-101. (in Chinese)

19. Plengvidhya N, Boonyasrisawat W, Chongjaroen $\mathrm{N}$, Jungtrakoon P, Sriussadaporn S, Vannaseang S, Banchuin N, Yenchitsomanus PT. Mutations of maturityonset diabetes of the young (MODY) genes in Thais with 
early-onset type 2 diabetes mellitus. Clin Endocrinol (Oxf). 2009; 70:847-853.

20. Holmkvist J, Almgren P, Lyssenko V, Lindgren CM, Eriksson KF, Isomaa B, Tuomi T, Nilsson P, Groop L. Common variants in maturity-onset diabetes of the young genes and future risk of type 2 diabetes. Diabetes. 2008; 57:1738-1744.

21. Lukasova P, Vcelak J, Vankova M, Vejrazkova D, Andelova K, Bendlova B. Screening of mutations and polymorphisms in the glucokinase gene in Czech diabetic and healthy control populations. Physiol Res. 2008; 57:S99-S108.

22. Marz W, Nauck M, Hoffmann MM, Nagel D, Boehm BO, Koenig W, Rothenbacher D, Winkelmann BR. G(30)A polymorphism in the pancreatic promoter of the glucokinase gene associated with angiographic coronary artery disease and type 2 diabetes mellitus. Circulation.
2004; 109:2844-2849.

23. Yang $S, D u Q$. Association of GCK -30G>a polymorphism with gestational diabetes mellitus and type 2 diabetes mellitus risk: A meta-analysis involving 18 case-control studies. Genet Test Mol Biomarkers. 2014; 18:289-298.

24. Nam JH, Lee HC, Kim YH, Cha BS, Song YD, Lim SK, Kim KR, Huh KB. Identification of glucokinase mutation in subjects with post-renal transplantation diabetes mellitus. Diabetes Res Clin Pract. 2000; 50:169176.

25. Ni X, Zhang J. Association between 9p21 genomic markers and ischemic stroke risk: Evidence based on 21 studies. PLoS One. 2014; 9:e9025

(Received October 6, 2015; Revised December 7, 2015; Accepted December 14, 2015) 